\title{
Resonance Near-Field Microwave Probing as a Method for Exploration of Deep Burn Wound Structures in Experiment
}

DOI: $10.17691 / \mathrm{stm} 2018.10 .3 .15$

Received November 12, 2017

A.K. Martusevich, DSc, Head of the Laboratory of Medical Biophysics, Institute of Traumatology and Orthopedics';

S.Yu. Krasnova, Junior Researcher, Laboratory of Medical Biophysics, Institute of Traumatology and Orthopedics 1 ; A.G. Galka, Junior Researcher, Laboratory of Medical Biophysics, Institute of Traumatology and Orthopedics'; Junior Researcher, Laboratory of Space Plasma Modeling2;

P.V. Peretyagin, Junior Researcher, Department of Experimental Medicine with Vivarium, Institute of Traumatology and Orthopedics';

A.V. Kostrov, DSc, Head of the Laboratory of Space Plasma Modeling ${ }^{2}$

${ }^{1}$ Privolzhsky Research Medical University, 10/1 Minin and Pozharsky Square, Nizhny Novgorod, 603005, Russia;

${ }^{2}$ Federal Research Center Institute of Applied Physics of the Russian Academy of Sciences, 46 Ulyanova St., Nizhny Novgorod, 603950, Russia

The aim of the investigation was to study the diagnostic value of the near-field microwave probing in the assessment of the normal and experimentally burned rat skin profiles.

Materials and Methods. The investigation was performed on 30 mature Wistar male rats divided into two groups of equal size. The animals of the control group $(n=15)$ did not undergo any manipulations except for a single microwave probing. A contact thermal burn covering $20 \%$ of the body area was modeled on the rats of the main group ( $n=15)$. Near-field microwave probing was carried out using a device designed in the Institute of Applied Physics of the Russian Academy of Sciences (Nizhny Novgorod, Russia) which enables the estimation of the object dielectric permittivity. A set of probes was used to assess dielectric characteristics of the skin at the depth of 2-5 mm.

Results. Real part of dielectric permittivity of the skin and subcutaneous structures in normal rats was determined to rise monotonously with the increase of the probing depth from 2 to $5 \mathrm{~mm}$ and a pitch of 0.5 to $1 \mathrm{~mm}$. The tissues of the burn wound demonstrate a higher level of real part of dielectric permittivity relative to the intact skin, the shift having different temporal dynamics. That is immediately after the burn application, changes of the parameter in the superficial biological tissue layers prevail and a day later they prevail in the deeper ones.

Conclusion. The method of the resonance microwave investigation can be used to monitor the structure of the skin in norm and in local changes including the depth of thermal tissue injuries.

Key words: microwave probing; dielectric properties of the skin; burns; dielectric permittivity.

\section{Introduction}

The overall prevalence and non-decreasing incidence of thermal traumas predetermine not only the development of innovative technologies of treatment of heavily burned patients but improvement of the diagnostic apparatus of combustiology [1-3]. At present, greatest importance for this cohort of suffers is a clinical assessment of the local status including the assessment connected with the use of a series of empirical algorithms for determining special characteristics of the received trauma (the rule of nines, etc.) [2-4]. At the same time, a great amount of diagnostic difficulties remain in combustiology connected with specifying precise borders of a burn injury, evaluation of the viability of tissues in the periwound zone, wound uniformity, and so on $[1,3,5]$. Separately stands the problem of verifying the injury depth of the skin and subcutaneous structures $[5,6]$.

To solve a variety of these tasks apart from the empiric approach prevailing in real clinical practice, the value of IR thermal imaging are being studied. This technology has been shown to be informative in some situations, however, it makes it possible to evaluate the state of the skin surface and the nearest underlying structures only. Modern options of ultrasound examination, though possessing high informativity and resolution in other pathology, fail to achieve the appropriate contrast in case of a thermal trauma [1, 5-7].

An additional difficulty encountered in tissue visualization in combustiology is a physical barrier (temporary and constant wound coatings) between the probe and the skin surface which is not always possible to remove for conducting diagnostic manipulations (for

Corresponding author: Andrey K. Martusevich, e-mail: cryst-mart@yandex.ru. 
example, when biocoatings containing matrices with stem cells are used) $[5,6,8,9]$. This is an obstacle for the majority of methods for examination of subsurface structures or integument, for US investigation in particular. All this inspired the researchers to seek and test fundamentally different technologies of profiling deep burn wounds and a periwound zone [8].

In this regard, a relatively new method of near-field resonance microwave profiling based on the study of tissue dielectric properties, i.e. dielectric permittivity and conductance, attracts special attention [8, 10-14]. This technique has been shown by previous investigations [15-17] to be highly informative in dermatology. It provides the possibility to make primary and differential diagnosing of various skin diseases and monitor the efficacy of the conducted treatment predicting the transition of the patient to the phase of remission. However the technique has not been previously used in combustiology.

The aim of the study was to study the diagnostic potentialities of near-field microwave probing in profiling a rat skin in norm and in the area of the experimentally burned wound.

\section{Materials and Methods}

The investigation was performed on 30 mature Wistar male rats divided into two groups of equal size. The animals of the control group $(n=15)$ did not undergo any manipulations except for a single microwave probing. A contact thermal burn covering $20 \%$ of the body area was modeled on the rats of the main group $(n=15)$ according to the proprietary methodology [18], and immediately after the injury and then $24 \mathrm{~h}$ later, the dielectric properties of the burn wound tissues have been studied.

All manipulations with the animals were carried out in compliance with the Guide for the Care and Use of Laboratory Animals (National Research Council, 2011); National standard of the Russian Federation 330442014 "Principles of Good Laboratory Practice"; ethic principles of the European Convention for the Protection of Vertebrate Animals used for Experimental and other Scientific Purposes (Strasbourg, 2006). The study protocol was approved by the Ethics Committee of the Privolzhsky Research Medical University.

Near-field microwave probing was carried out using a system, designed in the Institute of Applied Physics of the Russian Academy of Sciences (Nizhny Novgorod, Russia), and specialized software interfacing the system and computer, and enabling the calculation of real part of dielectric permittivity $[11,16]$. Skin dielectric characteristics were assessed at the depths of 2-5 mm using a series of probes. Measurements in all animals were taken at the same point localized in the middle part of the back on the preliminarily depilated surface. The results were processed using Statistica 6.0 software.

\section{Results and Discussion}

The results of the investigation showed the possibility of analyzing the dielectric properties of the rats skin in vivo by means of the examined device. The level of subsuperficial structure dielectric permittivity at the examined depths $(2-5 \mathrm{~mm})$ has been found to be less than 9 standard units and to monotonously rise with the increase of the probing depth (Figure 1).

Taking into account that each value is cumulative, i.e. shows the conductance of the entire subsuperficial layer up to the indicated depth (Figure 2), and the maximum parameter level is registered at a distance of $5 \mathrm{~mm}$ from the skin surface (see Figure 1), this is also reflected in the characteristic of the applied probing method as a near-field one.

The level of the examined parameter at the minimal and maximal depths differs by 2.55 times $(p<0.01)$ which is determined by the assessment of the deeper structures in the rats performing microwave profiling by the probe working at the depth of $5 \mathrm{~mm}$. It can be also explained by the fact that each subsequent dielectric permittivity value includes the previous one together with the contribution made by the tissues located in the

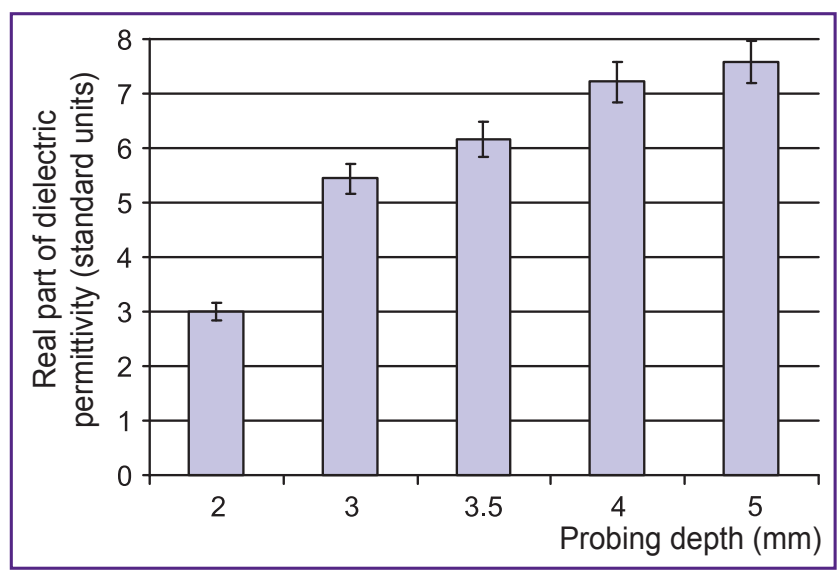

Figure 1. Dielectric permittivity profile of the skin and subcutaneous structures of healthy rats according to the data obtained by near-field microwave probing

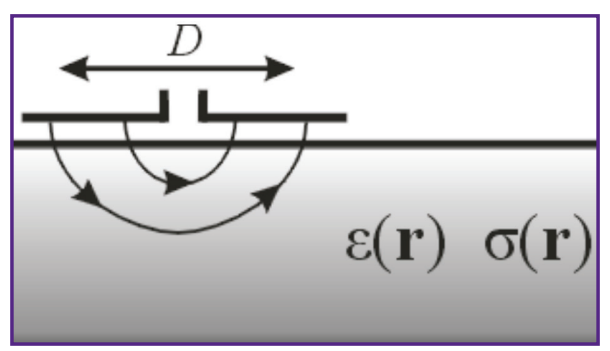

Figure 2. Schematic presentation of resonance near-field microwave probing of the subsuperficial structures $D$ - probe diameter; $\varepsilon(r)$ - dielectric permittivity of the medium being probed; $\sigma(r)$ - conductance of the medium being probed 
range from the previous to the current probing level [11, 19-21].

Based on the data obtained from the intact rats, a linear mathematical model of alterations in the dielectric permittivity of the animal skin has been built describing fully enough its subsuperficial profile (coefficient of determination - 0.91). Linear regression equation allowing prediction of the dielectric permittivity value at other probing depths is presented in the following form:

$$
y=1.5 x+0.455
$$

where $x$ is a probing depth $(\mathrm{mm}), y$ is the level of dielectric permittivity at a given depth (standard units).

This model can be applied to calculate physiological level of dielectric permittivity of the subsuperficial rat skin structures which may serve as a reference point for revealing its changes caused by various pathologic processes.

At the second stage, near-field microwave probing was used to study the dielectric properties of the skin and superficial tissue deep profiles in the animals with preliminarily induced thermal trauma (in the form of a contact thermal burn on the pre-depilated skin surface of the back). The dielectric permittivity of the subsuperficial tissues of the experimental burn wound was assessed immediately after the injury and a day after its modeling. It allowed the deep skin profiles to be formed by this parameter in the course of the experiment and in comparison with the intact biological tissue (Figure 3).

The dielectric parameters of the burn wound have been established to differ significantly from the properties of the intact tissue, these shifts being characterized by the growth of real part of the medium dielectric permittivity at both time points of observation. Thus, immediately after the thermal injury, the greatest changes have been noted in the nearest subsuperficial skin layers $(2-3 \mathrm{~mm})$ in which the examined parameter increases many times relative to the values in the intact rats (by 7.46 and 9.47 times at the probing depth of 2 and $3 \mathrm{~mm}$; $p<0.05$ for both cases) making about 24.0 and 25.8 standard units, respectively. It may be due to an intensive rapid local heating of the tissues at a small depth immediately after the exposure, whereas the deeper layers have not yet been involved in this process.

Later (a day after the injury), the depth profile of the skin dielectric permittivity transforms essentially (see Figure 3). In this period, deepening of the subsuperfical structure damage takes place which is accompanied by the decrease of the examined parameter at minimal depths $(2-3 \mathrm{~mm})$ with its growth relative to the intact skin at a distance of $3.5-5.0 \mathrm{~mm}$ below the skin surface $(p<0.05$ for all cases). The maximum of the dielectric permittivity was registered at the depth of $4 \mathrm{~mm}$ which corresponded to the zone of the most severe damage in our experiment. It speaks of a partial cooling of the superficial skin layers with simultaneous overheating of the most proximal ones and, consequently, shifting the

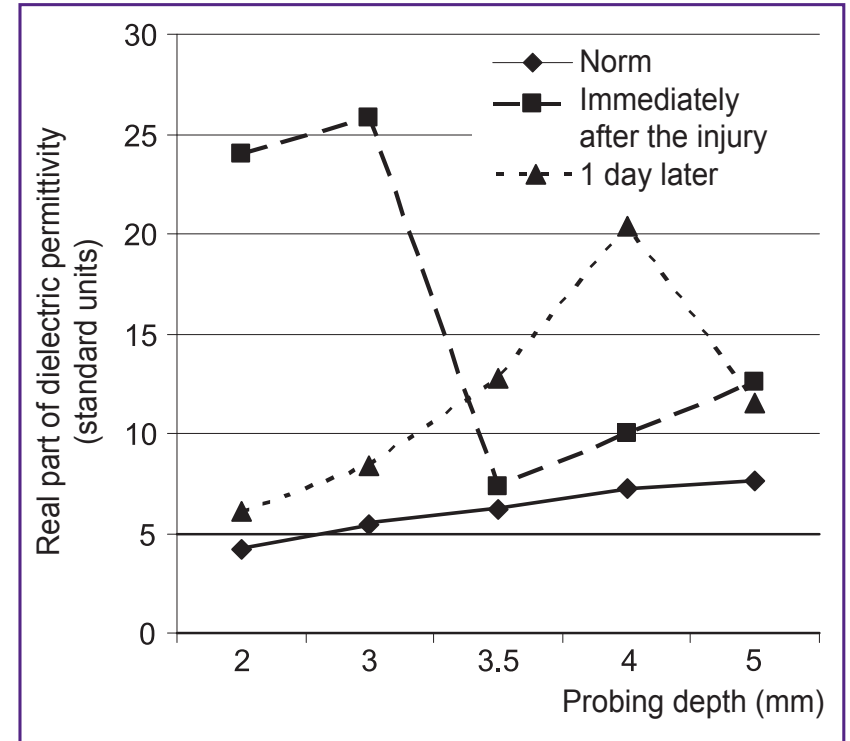

Figure 3. Dielectric permittivity profile of the skin and subcutaneous structures of the rats in norm and in experimentally-induced burn according to the data obtained by resonance near-field microwave probing

injury focus to the deeper layers. The data presented prove the possibility of monitoring the depths of the thermal tissue injuries and create an experimental basis for testing the processes of burn deepening in the posttraumatic period.

Thus, the investigations performed with the help of the near-field microwave probing made it possible to create a depth profile of the dielectric permittivity distribution for the skin of the healthy animals (rats of the Wistar line) which may serve as a physiological microwave pattern for the subsuperficial tissue study including various skin layers and the nearest subcutaneous structures. Real part of the skin dielectric permittivity was found to rise with the increase of the probing depth in the range of 2 to $5 \mathrm{~mm}$ with a pitch from 0.5 to $1 \mathrm{~mm}$. This allowed creation of the linear mathematical model of the depth structure of the examined parameter level distribution.

The model may help to predict the level of dielectric permittivity in deeper subsuperficial skin structures.

The tissues of the burn wound were found to demonstrate a higher level of real part of dielectric permittivity compared to the intact skin, these shifts having temporal dynamics: immediately after the thermal injury, changes of the parameter in the superficial biotissue layers prevail whereas a day later they prevail in the deeper ones.

\section{Conclusion}

The detected stability of the microwave profile enables the application of near-field resonance microwave probing of the skin for the assessment of its structure in norm and in local alterations (benign 
and malignant neoplasms, burns, etc.) and the derived equation may serve as a reference point for further studies of dielectric characteristics of the skin tissues in diverse experiments.

Research funding and conflict of interest. The study was not funded by any sources, and there are no conflicts of interest related to this study.

\section{References}

1. Peretyagin S.P., Dmitriev G.I., Aminev V.A., Levin G.Ya., Struchkov A.A., Kolesov S.N., Arefiev I.Yu., Atyasov I.N., Dokukina L.N., Khrulev S.E. The new medical technologies at the stage of preventive rehabilitation of the burnt patients. Medicinskij al'manah 2010; 2: 221-224.

2. Arai T. Burns. Nihon Rinsho 2016; 74(2): 231-235.

3. Daigeler A., Kapalschinski N., Lehnhardt M. Therapy of burns. Chirurg 2015; 86(4): 389-401, https://doi.org/10.1007/ s00104-014-2919-3.

4. Li H., Zhang J., Chen J., Song H., Liu Q., Fan X., Peng Y., Wu J. Integration of burn treatment and rehabilitation for a child with extremely severe burn. Zhonghua Shao Shang Za Zhi 2015; 31(2): 130-134.

5. Ida T., Iwazaki H., Kawaguchi Y., Kawauchi S., Ohkura T., Iwaya K., Tsuda H., Saitoh D., Sato S., Iwai T. Burn depth assessments by photoacoustic imaging and laser Doppler imaging. Wound Repair Regen 2016; 24(2): 349-355, https://doi.org/10.1111/wrr.12374.

6. Chin M.S., Babchenko O., Lujan-Hernandez J., Nobel L., Ignotz R., Lalikos J.F. hyperspectral imaging for burn depth assessment in an animal model. Plast Reconstr Surg Glob Open 2016; 3(12): e591, https://doi.org/10.1097/ gox.0000000000000558.

7. Kolesov S.N., Volovik M.G. Modern methodology for thermal-vision research and thermal-vision diagnostic apparatus. Journal of Optical Technology 2013; 80(6): 372378, https://doi.org/10.1364/jot.80.000372.

8. Bogomolova E.B., Martusevich A.K., Klemenova I.A., Yanin D.V., Galka A.G. Application of modern methods of visualization in study and prognosing of pathological scars. Meditsina 2017; 5(3): 58-75.

9. Turchin I.V. Methods of biomedical optical imaging: from subcellular structures to tissues and organs. PhysicsUspekhi 2016; 59(5): 487-501, https://doi.org/10.3367/ ufne.2015.12.037734.

10. Arsen'yev A.V., Volchenko A.N., Likhacheva L.V., Pecherskiy V.I. Close-field high frequency probing method in biological diagnostics. Nauchno-tekhnicheskiy vestnik informatsionnykh tekhnologiy mekhaniki $i$ optiki 2011; 2: 154-157.

11. Kostrov A.V., Smirnov A.I., Yanin D.V., Strikovsky A.V., Panteleeva G.A. Near-field microwave resonance diagnostics of inhomogeneous media. Bulletin of the Russian Academy of Sciences: Physics 2005; 69(12): 1911-1916.

12. Reznik A.N., Yurasova N.V. Near-field microwave tomography of biological objects technical physics. Technical Physics 2004; 49(4): 485-493, https://doi. org/10.1134/1.1736920.

13. Gaikovich K.P. Subsurface near-field scanning tomography. Phys Rev Lett 2007; 98(18): 183902, https://doi. org/10.1103/physrevlett.98.183902.

14. Hayashi Y., Miura N., Shinyashiki N., Yagihara S. Free water content and monitoring of healing processes of skin burns studied by microwave dielectric spectroscopy in vivo. Phys Med Biol 2005; 50(4): 599-612, https://doi. org/10.1088/0031-9155/50/4/003.

15. Raicu V., Kitagawa N., Irimajiri A. A quantitative approach to the dielectric properties of the skin. Phys Med Biol 2000; 45(2): L1-L4, https://doi.org/10.1088/0031-9155/45/2/101.

16. Kostrov A.V., Strikovskiy A.V., Yanin D.V., Smirnov A.I., Zagaynov V.E., Vasenin S.A., Druzhkova I.N., Panteleeva G.A., Davoyan Z.V. . Almanakh klinicheskoy meditsiny 2008; 17-2: 96-99.

17. Tamura T., Tenhunen M., Lahtinen T., Repo T., Schwan H.P. Modelling of the dielectric properties of normal and irradiated skin. Phys Med Biol 1994; 39(6): 927-936, https://doi.org/10.1088/0031-9155/39/6/001.

18. Peretyagin S.P., Martusevich A.K., Vazina I.R., Grishina A.A., Struchkov A.A., Luzan A.S., Kvitsinskaya N.A. The development of a new way of a combined burning injury modeling. Sovremennye tehnologii v medicine 2011; (2): 106109.

19. Reznik A.N., Yurasova N.V. Detection of contrast objects inside biological media by near-field microwave diagnostics. Technical Physics 2006; 51(1): 86-99, https://doi. org/10.1134/s1063784206010142.

20. Baloshin Y.A., Sorokin A.A., Volchenko A.N. Electrodynamic model of the near-field high frequency method of physical objects probing. Zvestiya vysshikh uchebnykh zavedeniy. Priborostroenie 2011; 54(12): 68-73.

21. Sunaga T., Ikehira H., Furukawa S., Shinkai $H$., Kobavashi H., Matsumoto Y., Yoshitome E., Obata T., Tanada S., Murata H., Sasaki Y. Measurement of the electrical properties of human skin and the variation among subjects with certain skin conditions. Phys Med Biol 2002; 47(1): N11-N15, https://doi.org/10.1088/0031-9155/47/1/402. 